\title{
Combined cutaneous findings with segmental odontomaxillary dysplasia: Review of the literature and proposal of a new clinical classification
}

\author{
Othman M Yassin' \\ Farouk B Rihani² \\ 'Consultant in pediatric dentistry, \\ Chairman of Pediatric Dentistry \\ Specialty at King Hussien Medical \\ Center, Royal Medical Services, \\ Amman, Jordan; ${ }^{2}$ Specialist in pediatric \\ dentistry, Prince Rashid Bin AL-Hassan \\ Hospital, Royal Medical Services, Irbid, \\ Jordan
}

\begin{abstract}
Segmental odontomaxillary dysplasia is characterized by variability of its clinical and radiological features, and may mimic other fibro-osseous lesions. We describe the case of a segmental odontomaxillary dysplasia in a 12-year-old male comprising of dermal, gingival, bony, and dental abnormalities. He presented with left midfacial diffuse hyperkeratotic erythematous lesion, ipsilateral hypopigmentation of upper lip with indistinct vermilion border, left-sided facial swelling, unilateral maxillary enlargement and ipsilateral failure of eruption of permanent teeth. Radiographic imaging showed abnormal bony trabeculation, impacted and missing teeth. Histopathologic findings showed characteristic features of segmental odontomaxillary dysplasia. We herein report a case of this rare unusual anomaly, review the literature, and propose a new clinical classification based on the limited number of previously reported cases in an attempt to categorize the clinical variants of the condition which might be helpful when treatment options are to be considered. Clinicians should be aware of its presence when encountering patients presenting with a facial cutaneous lesion especially when it is associated with facial asymmetry and unresponsive to treatment.
\end{abstract}

Keywords: hemimaxillofacial dysplasia, segmental odontomaxillary dysplasia, facial asymmetry, maxillofacial developmental anomaly

\section{Introduction}

Segmental odontomaxillary dysplasia (SOD) is a rare cause of nonprogressive unilateral facial asymmetry in children. ${ }^{1-3}$ It represents as unilateral maxillary developmental abnormality affecting the growth and maturation of bone, teeth and overlying gingival. ${ }^{4}$ SOD is characterized mainly by unilateral maxillary alveolar bone and gingival enlargement, ipsilateral dental abnormalities, distinctive radiographic bone trabeculation pattern, various degrees of facial asymmetry and in some cases variable facial cutaneous findings. ${ }^{1-5}$

\section{Case report}

A 12-year-old Arab healthy male was referred by a general dental practitioner to the pediatric dental clinic with a concerned parent about unerupted teeth and facial swelling. His family and medical history were unremarkable except for a left facial eczema-like lesion which was treated repeatedly by a dermatologist for the past three years without significant improvement. Extraoral examination revealed mild leftsided facial swelling with hyperkeratotic diffuse erythematous lesion involving the left midface and extending from the left infraorbital margin to the commisure of the lip. Hypopigmentation of the left and middle parts of the upper lip was evident with unidentifiable vermilion border (Figure 1).

Intraoral examination showed a dentition with all permanent teeth were present except the maxillary left permanent canine, and the maxillary left first and
Correspondence: Farouk B Rihani Irbid, AL-Huson, Postal Code 21510, P.O. Box 434, Jordan

Tel +962795689224

Fax+962 27060055

Email fbrihani@yahoo.com 


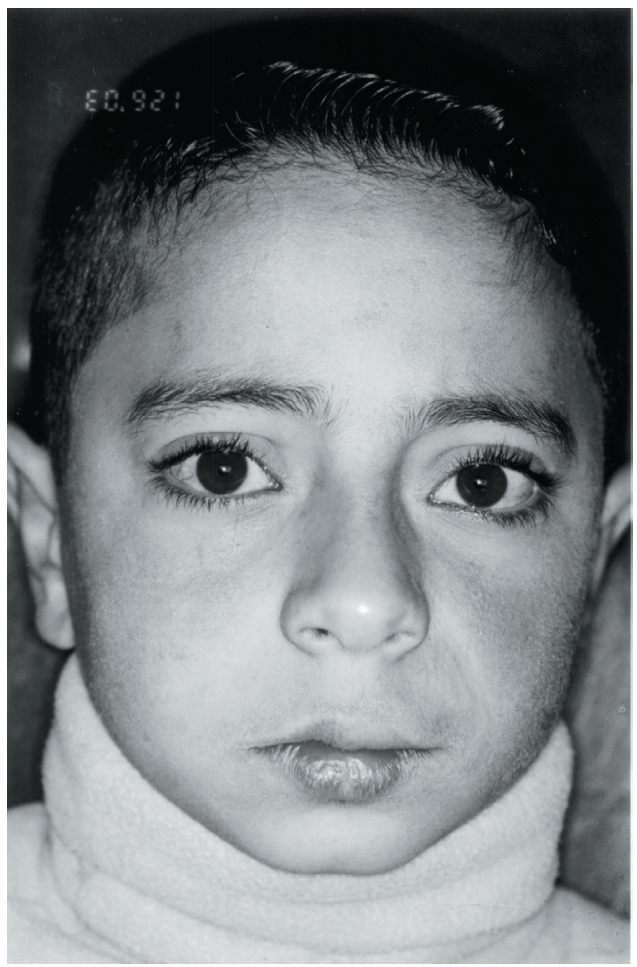

Figure I Frontal facial view of the patient with facial asymmetry and rough erythema involving left midface. Note hypopigmentation of upper lip and loss of vermilion border.

second premolars. The maxillary arch was asymmetric with enlarged alveolar bone buccolingually extending from the canine eminence to the maxillary tuberosity on the same side. The buccal gingival tissues covering the affected area was thickened and appeared fibrotic and partial obliteration of the labial sulcus was also noted (Figure 2).

An orthopantomogram showed irregular bone trabecular pattern of the left maxilla from the maxillary left permanent lateral incisor region to the ipsilateral maxillary tuberosity. The floor of the left maxillary sinus was partially elevated

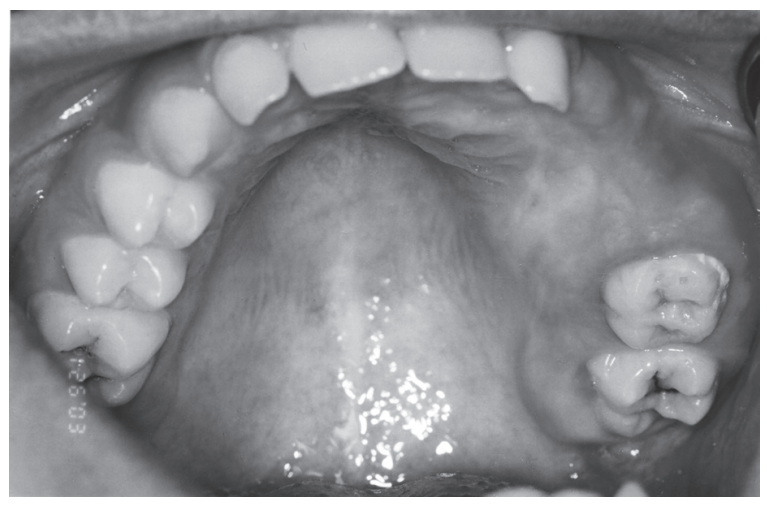

Figure 2 Intraoral view of the maxillary arch showing unilateral left buccolingual gingivo-alveolar enlargement with missing canine and premolars. when compared to the contralateral side. The maxillary left second premolar was missing and the maxillary left permanent canine was ectopically displaced. Other teeth within the lesion showed delayed or absent root development (Figure 3).

Bone biopsy after elevation of mucoperiosteal flap from the buccal maxillary left canine region was performed under general anesthesia. Histopathologic examination of the harvested bone sample revealed thick coarse bone trabculae of irregular and immature viable woven bone type with absence of lamellar bone and lacking osteoblastic/osteoclastic rimming. The bone marrow spaces were filled with active fine spindle cell stroma of fibrous connective tissue. Mitotic figures or pleomorphism were not observed. The histopathologic pattern was consistent with segmental odontomaxillary dysplasia (Figure 4).

\section{Literature review Clinical and radiographic features}

SOD is an unusual unilateral maxillary developmental anomaly with characteristic clinical, radiographic and histologic findings. Since its original description as hemimaxillofacial dysplasia by Miles and colleagues ${ }^{1}$ less than forty cases have been reported in the English literature. All cases reported appear to represent sporadic occurrence with no inheritance pattern. Males were affected more than females with a male-to-female ratio of $1.8: 1$. The condition commonly diagnosed in the first decade. The right and left sides of the maxilla are almost equally affected. ${ }^{1-12}$

Clinically SOD usually presents as a nonprogressive facial asymmetry, ipsilateral gingivo-dento-alveolar maxillary involvement from the canine eminence anteriorly until the maxillary tuberosity posteriorly, and with or without facial cutaneous lesions. Radiographically SOD shows

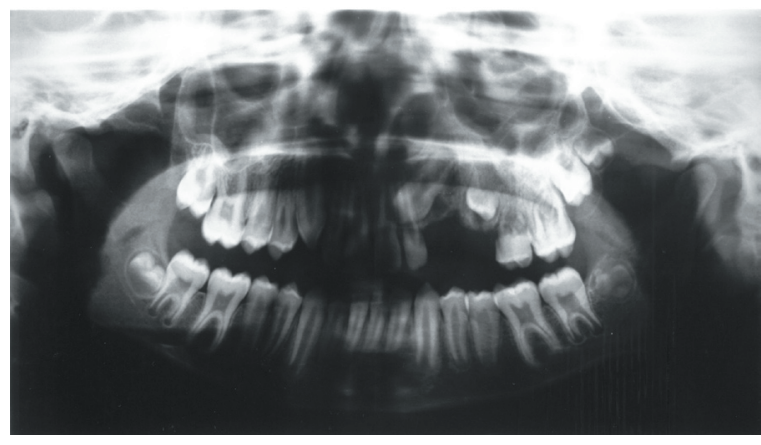

Figure 3 Orthopantomogram showing radiopacity with irregular bone trabecular pattern distal to maxillary left permanent canine. Note also reduction of size of ipsilateral maxillary sinus, absent left second premolar and abnormal root morphology of left deciduous and permanent molars. 
$\bar{a}$

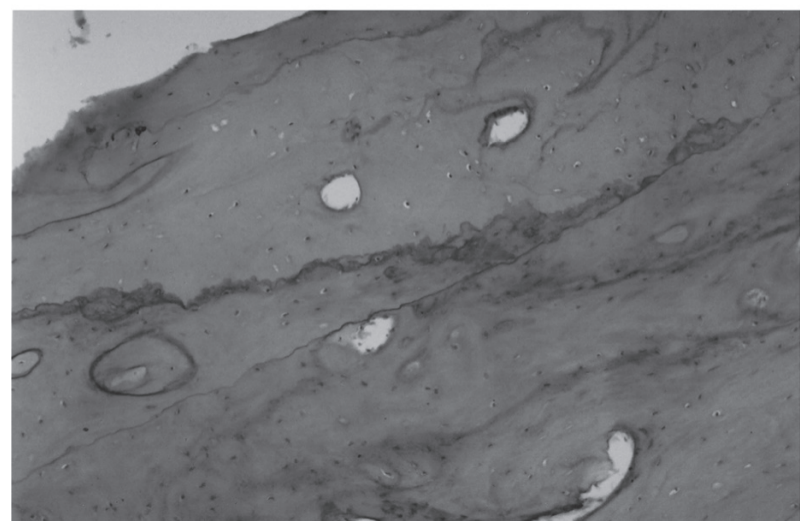

Figure 4A Photomicrograph of bone biopsy of the buccal maxillary left canine region shows the characteristic features of segmental odontomaxillary dysplasia with thick trabeculae of viable woven bone, absence of cortical bone, and prominent irregular resting/reversal lines (hematoxylin and eosin stain). Original magnification $\times 100$.

mainly bony sclerosis with thickened and coarse irregular bone trabeculation pattern producing an ill-defined opacity. ${ }^{2,3,6,7}$ Clinical and radiological features of SOD are shown in Table $1 .{ }^{1-12}$

Facial cutaneous manifestations of SOD are variable and affect up to $48 \%$ of SOD patients of whom $80 \%$ are males. ${ }^{1-12}$ The most common facial cutaneous manifestations are hypertrichosis followed by facial erythema, lip hypopigmentation, and Becker's nevus. ${ }^{1,3,5,6,8,12}$ Other cutaneous manifestations include "hairy nevus", discontinuity of the vermilion border, depression of the cheek, and facial hyperpigmentation. ${ }^{5,7,12}$ When hypertrichosis is the associated cutaneous lesion, it is only limited to males. ${ }^{1,5,8,12}$

\section{Etiology and pathogenesis}

The etiopathogenesis of SOD is unknown, although it is suggested that it might be due to a localized developmental $\bar{b}$

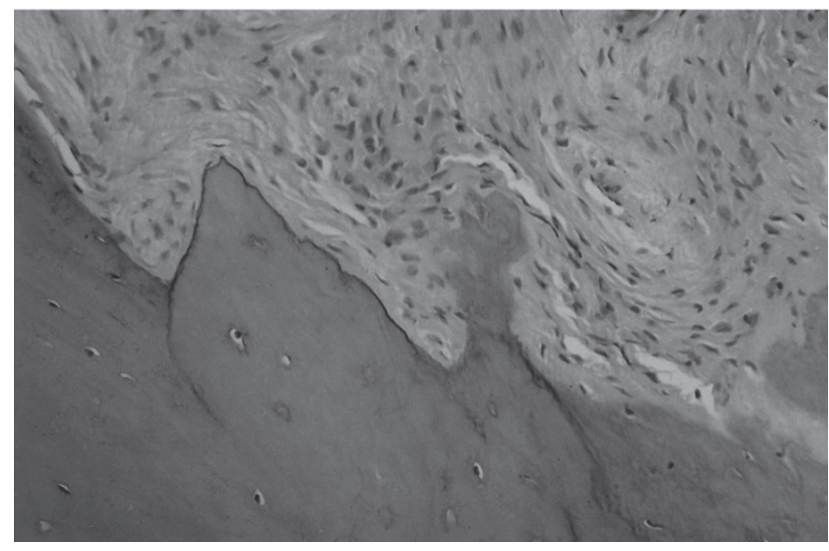

Figure 4B Higher magnification with uninflamed fibrous stroma and lack of osteoblastic/ osteoclastic rimming (hematoxylin and eosin stain). Original magnification $\times 200$.

abnormality in utero, ${ }^{2}$ a unilateral developmental field defect involving anlagen of the first and second branchial arches, ${ }^{5}$ or a postzygotic mutation or a similar phenomenon affecting a progenitor cell and could have resulted in an altered clone of cells that influenced the morphogenesis of the ecto-mesodermal tissues in a segment of the maxilla and the overlying facial tissues. ${ }^{8} \mathrm{~A}$ viral or bacterial infection of the maxillary division branches of the trigeminal nerve have been proposed as initial causative factors in SOD. ${ }^{3}$

\section{Histopathology}

Histologic characteristics of the anomaly are nonspecific noninflammatory connective tissue thickening and fibrosis of gingival tissues. ${ }^{1,2}$ Alveolar bone shows absence of mature lamellar bone, presence of thick trabeculae of immature woven bone with prominent resting/reversal lines, uninflamed fibrous stroma, and lack of osteoblastic and

Table I Relative frequency of clinical and radiographic characteristics of SOD

\begin{tabular}{lll}
\hline $\mathbf{7 5} \%-100 \%$ & $\mathbf{5 0 \% - 7 5 \%}$ & $\mathbf{2 5 \% - 5 0 \%}$ \\
\hline Mild-to-moderate facial asymmetry & Nonprogressive growth & Cutaneous facial lesion \\
$\begin{array}{l}\text { Buccolingual alveolar and gingival } \\
\text { thickening }\end{array}$ & Diagnosis in first decade & Splayed roots \\
Hypodontia & Affected male & Reduced size of pulp chambers \\
Widely spaced/displaced teeth & Hypoplastic teeth & Large pulp chambers \\
Ill-defined radiopacity & Increased mesiodistal crown dimension & \\
Vertically oriented bone trabeculation & Delayed eruption/impaction of teeth & \\
Thickened bone trabeculation & Root resorption/ill-defined root & \\
Reduced size of ipsilateral maxillary & morphology & \\
sinus &
\end{tabular}

Abbreviation: SOD, segmental odontomaxillary dysplasia. 
osteoclastic activity. ${ }^{1-4,6}$ Dental defects include circumpulpal dentin tubular defects, irregular pulp/dentin interface with pseudoinclusions, focal deficiency in odontoblastic layer and coarse fibrous pulp with pulp stones. ${ }^{11}$

\section{Diagnosis}

SOD may go unrecognized or misdiagnosed. ${ }^{6,12}$ Differential diagnosis includes monostotic fibrous dysplasia, regional odontodysplasia, gingival fibromatosis, segmental hemifacial hypertrophy, and various benign odontogenic tumors..$^{3,4,6,12}$ Clinical and radiological similarities between SOD and these lesions exist, which makes histopathologic examination necessary to accurately establish the diagnosis and exclude other pathological entities.

\section{Management}

Treatment modalities for SOD are still insufficient. Due to the nonprogressive nature and the nonsevere form of facial asymmetry in SOD recontouring osteotomy is not advised. Treatment can only be limited to restoring the functional dental occlusion in the affected side by orthodontic tooth movement and/or prosthetic replacement of missing teeth, and should be delayed until the pubertal growth spurt has ended. ${ }^{4,9}$

\section{Proposed classification}

The clinical variability of extraoral cutaneous manifestations of SOD necessitates a detailed clinical classification which can encompass all previously reported and future clinical variants of the condition. Furthermore, this classification should be simple to implement, useful during clinical evaluation procedure, and helpful in establishing general treatment guidelines for the different types of SOD. The classification proposed here fulfills these criteria, and intended to categorize and distinguish the clinical variants that could occur in SOD. This categorization mainly differentiates between SOD clinical variants according to the presence or absence of a facial cutaneous lesion. The classification described here not only subcategorized these cutaneous lesions according to the type of the lesion but also utilizes a combination system to further subclassify these combined cutaneous lesions when they occur in binary, tertiary or even quaternary combinations.

Therefore, according to previously reported cases of SOD, ${ }^{1-12}$ we propose a new clinical classification of SOD based on the presence and type of facial cutaneous manifestations. We recognize two major types: type I classical with gingivo-dento-alveolar manifestations without extraoral facial involvement, ${ }^{1,2,7-11}$ and type II cutaneous with

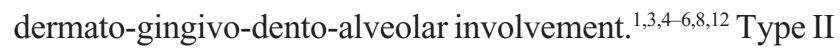
is further differentiated into four subtypes: type IIa hypertrichotic, ${ }^{1,5}$ type IIb pigmentory, ${ }^{3}$ type IIc erythematous, ${ }^{8}$ and type IId commissural defect. ${ }^{4}$ Furthermore, combinations between type II subtypes are possible such as type IIab with hypertrichotic-pigmentory combination, ${ }^{6,12}$ type IIabd with hypertrichotic-pigmentory-commissural defect combination, ${ }^{5}$ and type IIbc in our patient with pigmentory-erythematous combination.

\section{Conclusion}

SOD is characterized by variability of its clinical and radiological features, and may mimic other fibro-osseous lesions. Therefore, the definitive diagnosis of this lesion remains dependent on histological evaluation. Almost half of patients with SOD present with a facial cutaneous lesion which could be hypertrichotic, pigmentory, erythematous, commissural defect, or combination of these. This puts dermatologists in an ideal place to identify such patients, and as gingivo-dentoalveolar involvement is constant in all cases of SOD, the role of dentists is also crucial in early diagnosis. Early recognition of SOD also requires appropriate referrals to medical colleagues and interdisciplinary team approach mandatory, so patients with SOD can gain access to specialist dental care, including orthodontics, prosthodontics, and oral surgery.

Although SOD is a rare entity, clinicians should be aware of its presence when encountering patients presenting with facial asymmetry especially when it is associated with a facial cutaneous lesion, and should also consider it in differential diagnosis when treating a facial cutaneous lesion associated with facial asymmetry and unresponsive to treatment.

\section{Disclosure}

The authors report no conflicts of interest in this work.

\section{References}

1. Miles DA, Lovas JL, Cohen MM Jr. Hemimaxillofacial dysplasia: a newly recognized disorder of facial asymmetry, hypertrichosis of the facial skin, unilateral enlargement of the maxilla, and hypoplastic teeth in two patients. Oral Surg Oral Med Oral Pathol. 1987;64:445-448.

2. Danforth RA, Melrose RJ, Abrams AM, et al. Segmental odontomaxillary dysplasia. Report of eight cases and comparison with hemimaxillofacial dysplasia. Oral Surg Oral Med Oral Pathol. 1990;70:81-85.

3. Becktor KB, Reibel J, Vedel B, et al. Segmental odontomaxillary dysplasia: clinical, radiological and histological aspects of four cases. Oral Dis. 2002;8:106-110.

4. Prusack N, Pringle G, Scotti V, et al. Segmental odontomaxillary dysplasia. A case report and review of the literature. Oral Surg Oral Med Oral Pathol Oral Radiol Endod. 2000;90:483-488.

5. Paticoff K, Marion RW, Shprintzen RJ, et al. Hemimaxillofacial dysplasia. A report of two new cases and further delineation of the disorder. Oral Surg Oral Med Oral Pathol Oral Radiol Endod. 1997;83:484-488. 
6. DeSalvo MS, Copete MA, Riesenberger RE, et al. Segmental odontomaxillary dysplasia (hemimaxillofacial dysplasia): case report Pediatr Dent. 1996;18:154-156.

7. Packota GV, Pharoah MJ, Petrikowski CG. Radiographic features of segmental odontomaxillary dysplasia. A study of 12 cases. Oral Surg Oral Med Oral Pathol Oral Radiol Endod. 1996;82:577-584.

8. Jones AC, Ford MJ. Simultaneous occurrence of segmental odontomaxillary dysplasia and Becker's nevus. J Oral Maxillofac Surg. 1999;57:1251-1254.
9. Drake DL. Segmental odontomaxillary dysplasia: an unusual orthodontic challenge. Am J Orthod Dentofacial Orthop. 2003;123:84-86.

10. Gavalda C. Segmental odontomaxillary dysplasia. Med Oral. 2004;9:181.

11. Armstrong C, Napier SS, Boyd RC, et al. Histopathology of the teeth in segmental odontomaxillary dysplasia: new findings. J Oral Pathol Med. 2004;33:246-248.

12. Welsch MJ, Stein SL. A syndrome of hemimaxillary enlargement, asymmetry of the face, tooth abnormalities, and skin findings (HATS) Pediatr Dermatol. 2004;21:448-451. 
